\title{
15
}

\section{Incoming Call Screening (ICS) service}

\author{
P. Bleuse, B. Vilain \\ Alcatel Telecom \\ Switching Systems Division \\ Network Systems and Product Management \\ 10, rue Latécoère, B.P. 57 \\ F-78141 Vélizy Cedex, France \\ Tel: $\quad+33(0) 130779665$ \\ Fax: $+33(0) 130779886$ \\ bernard.vilain@vz.cit.alcatel.fr
}

\begin{abstract}
For new telephone companies to position themselves efficiently and cost effectively as long-distance network operators (LDNOs), they need a service to assist them in recognizing and screening local-loop subscribers placing a longdistance or international call.

They must be able to:

- Check that a caller, identified by the calling line identity (CLI) and intending to use their network, is registered.

- Check that they have the information necessary to bill the call.

- Handle calls from unregistered callers and route them selectively towards attendants. Attendants work for operators or telephone companies and are in charge of new and existing subscribers. Attendants in a call center must be able to propose a new intelligent-network (IN) service subscription to these unregistered callers.
\end{abstract}

- Reroute a call meeting a busy or no-reply condition.

- Restrict calls based on the CLI and the dialed number.

- Generate a Call Detail Record (CDR) for billing purposes. 
Alcatel proposes the above capabilities to these long-distance network operators (LDNOs) with its new Incoming Call Screening (ICS) service. The ICS service will allow them to check if an incoming call request has been generated by a subscribed user or not, to validate such a request, and to process it accordingly.

The service is invoked whenever a long-distance call attempt is received on incoming trunks, belonging to one or several predefined trunk categories, and when the caller has selected the LDNO as the carrier through equal-access mechanisms.

Thus, the core of Alcatel's ICS service is its ability to check incoming call requests and decide either on further routing or on rejection. Performing a check of this type inside the operator's network makes use of IN architecture, and both the checking and call-handling actions can be fairly sophisticated.

This article contains information on these actions, including the concept of a caller profile, service logics, service triggering, service provision, service subscription, and statistics. The appendix contains a complete glossary and list of abbreviations.

\section{Keywords}

Service creation, service triggering, service subscription, call screening, call billing, long-distance network operators

\section{ICS SERVICE OVERVIEW}

This document describes the Intelligent Network (IN) Incoming Call Screening (ICS) service. The ICS service allows the served network operator or the longdistance network operator (LDNO) to check if an incoming call request has been generated by a subscribed user or not, to validate such a request, and to process it accordingly.

As shown in the figure below, LDNOs do not usually have subscribers connected to them locally. Subscribers place calls through the local-loop operator to the long-distance network operator or international carrier network. The architecture shown below does not include Incoming Call Screening (ICS) or any intelligent-network platform. 


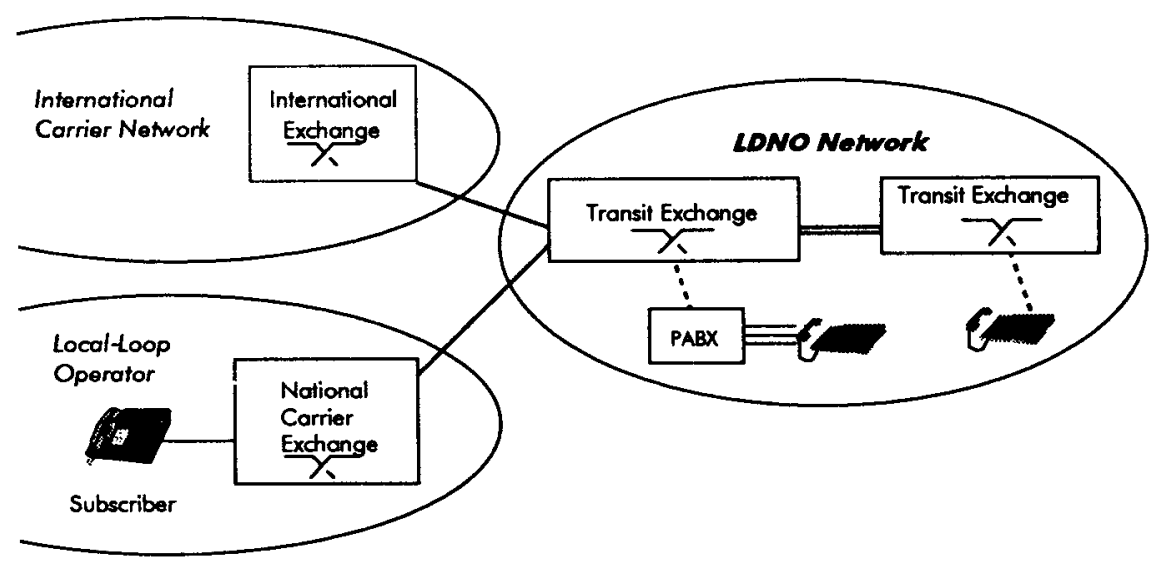

Figure 1 Types of call and network without intelligent network platform.

The next figure shows the same architecture as above, with the difference that it includes an intelligent-network platform supporting IN services, in this case, the ICS service.

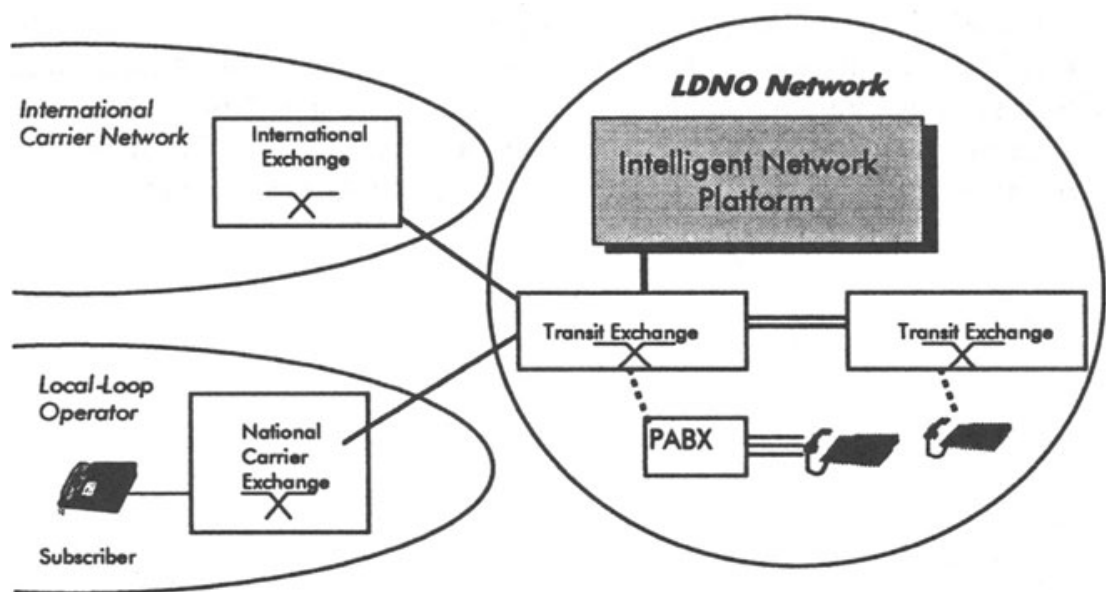

Figure 2 Types of call and network with an intelligent-network platform and Alcatel's Incoming Call Screening (ICS) service. 
Alcatel's new Incoming Call Screening service lets LDNOs:

- Check that a caller, identified by the calling line identity (CLI) and intending to use their network, is registered.

- Check that they have the information necessary to bill the call.

- Handle calls from unregistered callers and route them selectively towards attendants. Attendants work for operators or telephone companies and are in charge of new and existing subscribers. Attendants in a call center must be able to propose a new intelligent-network (IN) service subscription to these unregistered callers.

- Reroute a call meeting a busy or no-reply condition.

- Restrict calls based on the CLI and the dialed number.

- Generate a Call Detail Record (CDR) for billing purposes.

According to the agreement with the network operator, the types of call and network of the ICS service are listed below.

- The origin network can be an LDNO or a national carrier.

- The transit network is an LDNO.

- The destination network can be an LDNO, a national carrier, or an international carrier.

Inside the LDNO network, a service user can invoke an IN service. The call is routed to the Service Switching Function (SSF) via PSTN/ISDN/PLMN. The SSF will invoke the service for this particular call at the Service Control Point (SCP). The SCP performs translation and instructs SSF for call completion. The Service Management Point (SMP) is used for the service management. The Information System (IS) enables the service provider to manage all data related to the service. 


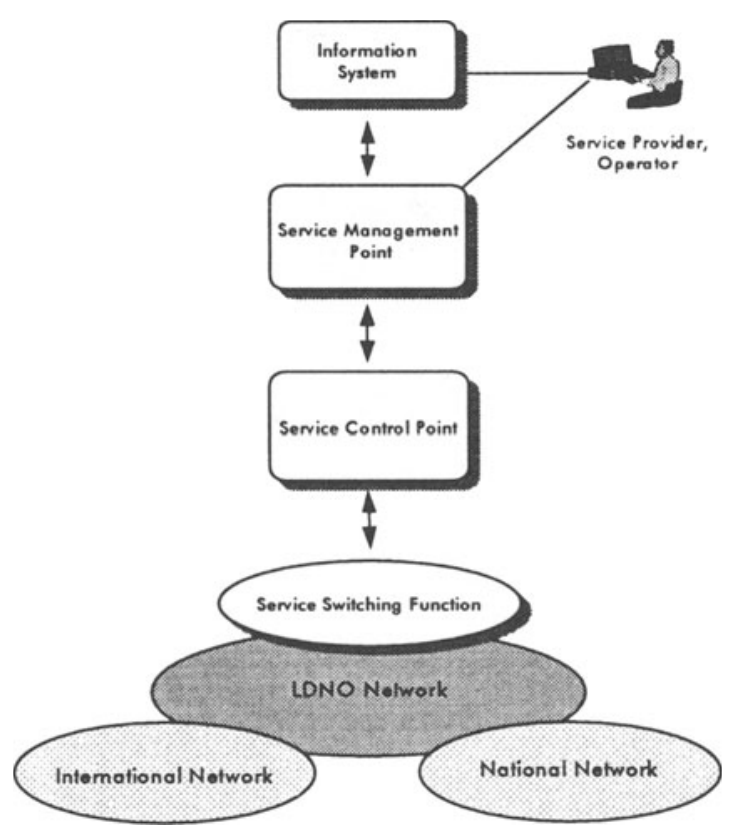

Figure 3 Invoking the ICS service inside the LDNO network.

The Incoming Call Screening service includes the following features:

- Call routing, in which the call is routed to an announcement or to an E164 address.

- Service activation, achieved by specifying the subscription start and expiration dates.

- National-to-national, and national-to-international calling capabilities.

- Service unavailability, which means that if the ICS service is unavailable, whatever the reason, the calls are routed to the dialed number. The call data record includes the information "unchecked call".

- Routing to an interactive voice response server.

- Routing to a voice-mail delivery system.

- Playing an announcement to a party. 
- Implementation of a call center overload mechanism to avoid congestion at the attendant's call center.

- Implementation of operator-defined limitation on destination; the list of restricted destinations is common to all caller profiles.

- Implementation of subscriber-defined limitation on destination; the list of restricted destinations applies to a given CallerID; the operator-defined restrictions take precedence over the subscriber-defined restrictions.

The following sections describe the concept of a caller profile, ICS service logic, service triggering, service provision, service subscription, and statistics.

\section{CONCEPT OF A CALLER PROFILE}

For the ICS service, the interface between the network and the information system is of utmost importance. The Commercial Information System (IS-C) manages the mapping between the caller identification and the caller profile, and, through the Technical Information System (IS-T), passes this information to the network. The network handles each call along the lines of a predefined set of service logics.

\subsection{Caller identification, the CallerID}

The caller identification, also referred to as the CallerID, is composed of:

- the LineID, including the line identities as received in the signaling; the line identities are:

- the Calling Line Identity (CLI) provided and certified by the network,

- the User-Provided Identity (UPI);

- the calling party category (CPC) provided by the network;

- the access prefix composed by the caller and transported by the network;

- any additional identity provided by the signaling system along with the calling line identity (CLI); the CLI is provided and certified by the network.

\subsection{Caller profile}

The caller profile is composed of two information elements:

1. The subscription profile which reflects the situation of the subscriber vis-à-vis the subscription process.

2. The back-office profile which provides additional characterization of the caller. 
The subscription profile reflects the situation of the subscriber vis-à-vis the subscription process. Types of subscription status or situation are designated as unknown, undesired, prospect, target, subscriber, nomadic, suspended, and withdrawn.

The back-office profile provides additional characterization of the caller. This information is transparent to the network. The network transmits the information to the IS-T via the Call Detailed Record (CDR).

\subsection{Modifying the caller profile}

Each modification of the caller profile associated with a CallerID is passed from the IS-C to the IS-T and then to the network. There, it is transformed into a service logic and a group of settings, which are then entered into the network database. Thus, caller profiles are not used as such by the network which can only address the service logic associated with a given caller profile. When a call is processed by the ICS service, the network deduces a service logic from the CallerID. The service logic applied to the caller profile (for example, authorization of routing to a dialed number) is completed with settings applied to the given CallerID.

\subsection{Associating CallerIDs to caller profiles}

The association of a CallerID to a caller profile is received from the information system. Association with the service logic to be used is determined by the caller profile and by the call character "direct".

With the ICS service, the operator has the ability to specify the CallerID-tocaller profile relationship, either by:

- Specifying a global association with a Calling Party Category (CPC); this would be useful in defining the processing of calls from the lines with the "priority" or "payphone" category, for example. This type of association is managed by the network; or by

- Indicating a complete CallerID; this type of association is managed by the information system.

The figure below shows the principle of determining the caller profile from the CallerID. 


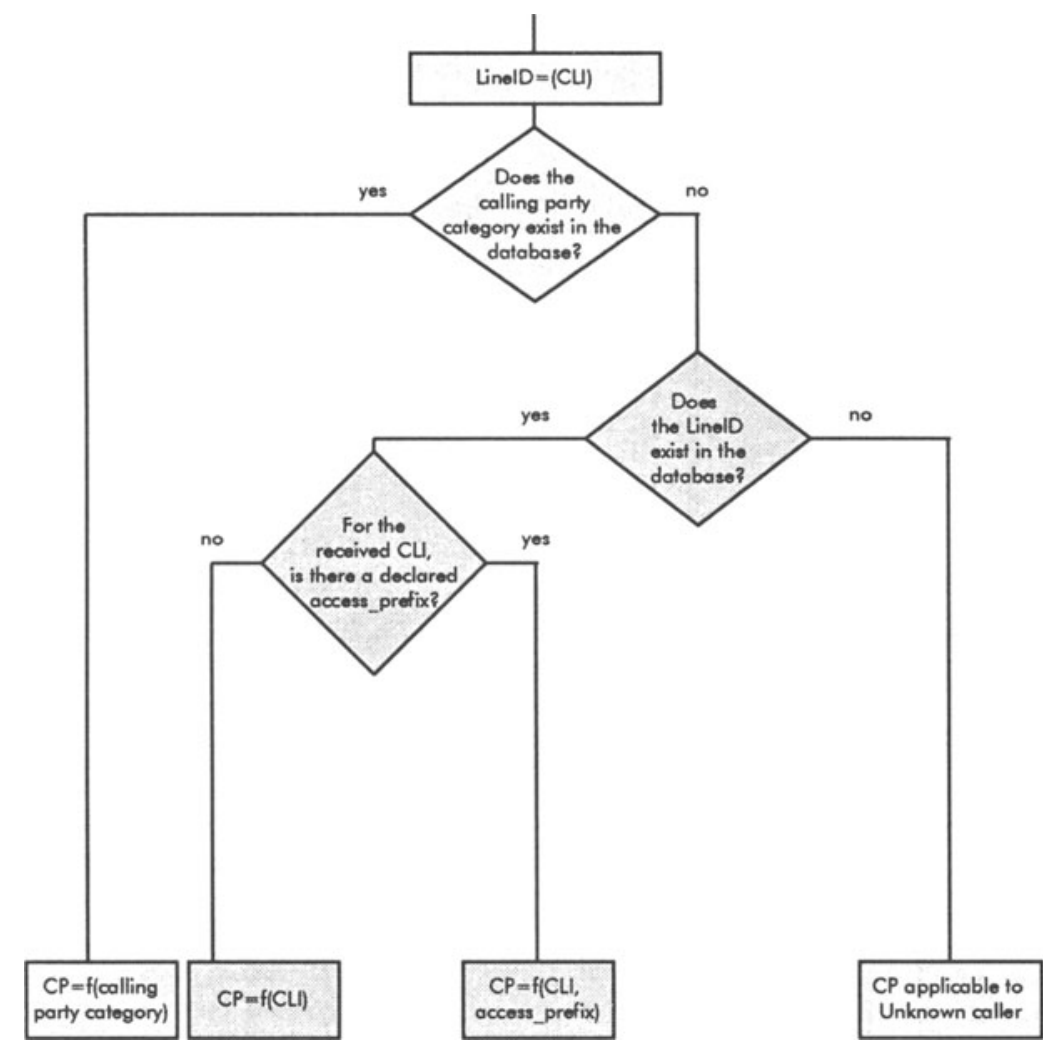

Figure 4 Principle of determining the caller profile from the CallerID.

\subsection{Handling caller profiles}

The caller profiles are handled as follows:

Neither the IS-C nor the IS-T knows the service logics associated with the caller profile. When an association between a caller profile and a set of CallerIDs is received by the network from the IS-C through the IS-T, the given caller profile is converted into a service logic applicable by the network to the calls from the set of CallerIDs. An example would be the downloading of a prospect's or a target's file.

After processing a call, the related caller profile is conveyed by the network down to the CDR where it is registered. The processing of the CDRs by the IS-C may imply a change in the caller profile. This change is sent back to the network, almost in real time. An example would be the IS-C-managed "restriction on 
credit overflow". Once the IS-C detects a floor overflow for example, a message requiring the modification of the CallerID is sent to the network.

The "unknown" caller profile is not explicitly declared, and the associated service logic is used when the CallerID is not found in the network database. The network is not allowed to change a caller profile on its own initiative; if such a change is necessary, it is always decided upon and ordered from the IS-C. From a logical point of view, the network is not allowed to create caller-related data on its own initiative. The creation of such data is allowed only if the service logic associated with the caller profile includes this data handling.

\section{ICS SERVICE LOGIC}

The ICS service offers identified call handling procedures as service logics. A service logic program is associated with a number of calling parties, via the relevant (set of) caller profile(s), and executed in the event of triggering the service by a call placed by one of these calling parties. For a given caller, there will be one given associated caller profile, which in turn determines one given service logic, with a set of given values to be applied to the call.

\subsection{Determining the service logic}

Each CallerID has a caller profile. The caller profile determines the service logic to be applied. The caller profile determines a group of global settings, applicable to all CallerIDs having that caller profile. The CallerID can also determine a group of specific settings, applicable to the CallerID, when can then override the global settings. The service logic consults the two groups of settings and takes them into account in the service logic. 


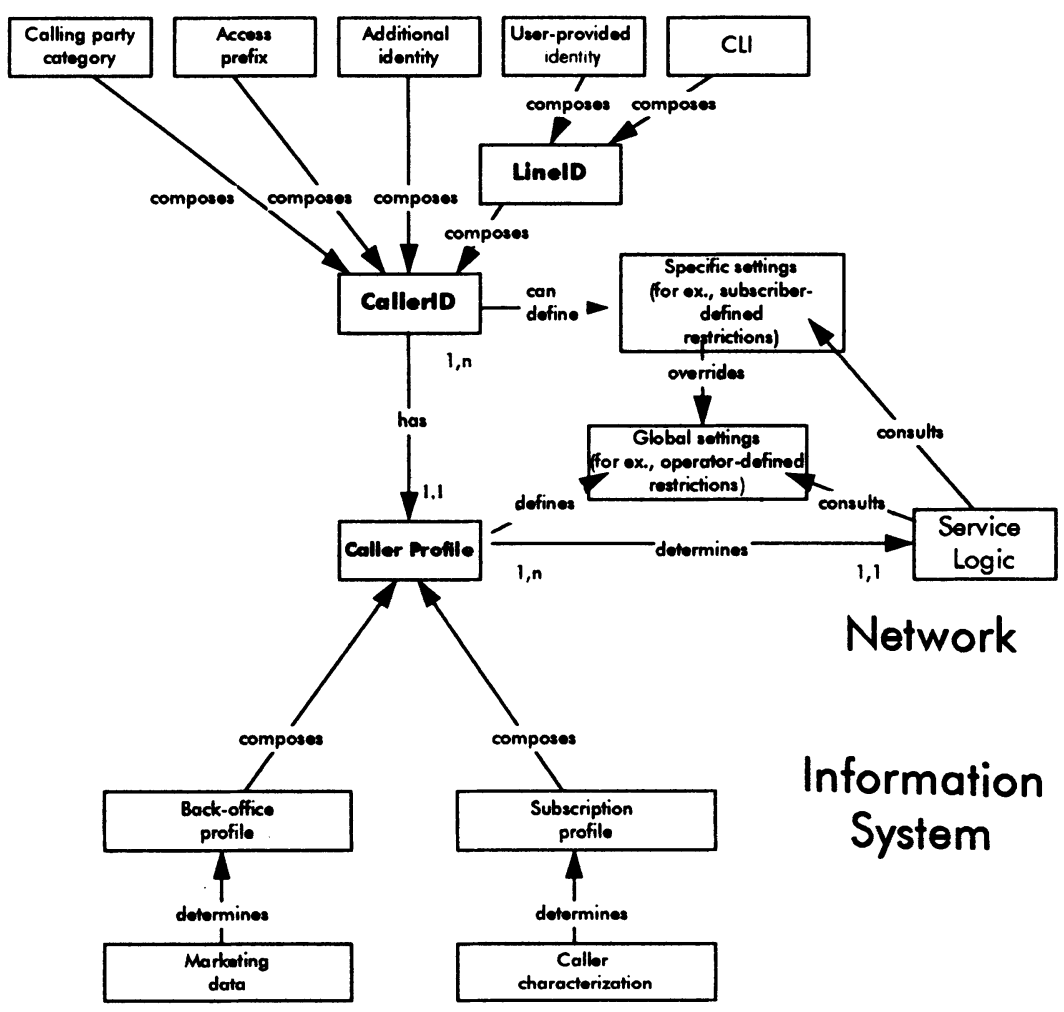

Figure 5 How the CallerID and the caller profile interact to determine the service logic.

To be successful, operators need to apprehend a wider range of caller profiles, beyond the rather simplistic distinction between "registered callers" and "unregistered callers".

Mapping between the CallerIDs and the caller profiles is determined by the Commercial Information System (IC-S) and not by the network. The mapping decision is based on information received from several sources, including market studies (prospect lists), the attendants' system (turning prospects into registered subscribers), and the network. 


\subsection{Service logic functions}

The service logic comprises the following elementary functions, and maximum flexibility is supported as to their sequencing and combination in a more or less complex structure:

- Routing the call to a given destination (this can be the called party, a voicemessaging system, an attendant position).

- Sending a verbal announcement to the caller as a final destination, for example, in the event of failure or a rejected call.

- Creating a Call Detailed Record (CDR), with all the necessary details of the call.

- Checking if a busy or no-reply condition is encountered.

- Checking if operator-defined, originating-call barring restrictions apply to the caller profile.

- Checking if subscriber-defined, originating-call barring restrictions apply to the CallerID.

- Forcing a network-originated call release.

- Sending a subsequent verbal announcement to the caller.

- Checking against call filtering, if applicable to the routing address.

\subsection{Service logic routing actions}

A service logic identifies a set of actions to be performed in various service logic situations (such as a busy condition). The basic principle consists in using the alternative destination routing of the first determined destination in the event of the busy condition. Thus, the service logic is capable of determining several levels of alternative actions. For example, a call, primarily routed to attendants, can be routed to the dialed number if the attendants are busy, then rerouted to an operator-managed voice-mail delivery system in case the dialed number is busy.

Therefore, the basic principle consists in using alternative destination routing in the event that the first determined destination is busy. The various service logic routing actions are given in the table below. 
Table 1 Types of service logic routing actions

\begin{tabular}{|c|c|c|c|}
\hline $\begin{array}{l}\text { Applicable action } \rightarrow \\
\boldsymbol{V}_{\text {Type of routing }}\end{array}$ & $\begin{array}{l}\text { Call center } \\
\text { overload }\end{array}$ & $\begin{array}{l}\text { Alternative } \\
\text { destination on } \\
\text { no reply }\end{array}$ & $\begin{array}{l}\text { Alternative } \\
\text { destination on } \\
\text { busy }\end{array}$ \\
\hline \multicolumn{4}{|l|}{ To an announcement } \\
\hline $\begin{array}{l}\text { Routing to an } \\
\text { announcement }\end{array}$ & $\begin{array}{l}\text { Not } \\
\text { applicable }\end{array}$ & Not applicable & $\begin{array}{l}\text { Not } \\
\text { applicable }\end{array}$ \\
\hline \multicolumn{4}{|l|}{ To attendants } \\
\hline $\begin{array}{l}\text { Normal routing to the } \\
\text { attendants }\end{array}$ & Applicable & Applicable & Applicable \\
\hline $\begin{array}{l}\text { Priority routing to the } \\
\text { attendants }\end{array}$ & $\begin{array}{l}\text { Not } \\
\text { applicable }\end{array}$ & Applicable & Applicable \\
\hline
\end{tabular}

To a network address

\begin{tabular}{l|l|ll}
\hline $\begin{array}{l}\text { Routing to the dialed } \\
\text { number }\end{array}$ & $\begin{array}{l}\text { Not } \\
\text { applicable }\end{array}$ & $\begin{array}{l}\text { Conditional } \\
\text { (subject to caller } \\
\text { acceptance) }\end{array}$ & Applicable \\
$\begin{array}{l}\text { Routing to an operator- } \\
\text { managed IVR server }\end{array}$ & $\begin{array}{l}\text { Not } \\
\text { applicable }\end{array}$ & Applicable & Applicable \\
$\begin{array}{l}\text { Routing to an operator- } \\
\text { managed voice-mail } \\
\text { delivery system } \\
\text { (not applicable as initial } \\
\text { routing) }\end{array}$ & $\begin{array}{l}\text { Not } \\
\text { applicable }\end{array}$ & Applicable & Applicable \\
$\begin{array}{l}\text { Routing to another } \\
\text { network address }\end{array}$ & $\begin{array}{l}\text { Not } \\
\text { applicable }\end{array}$ & Applicable & Applicable \\
\hline
\end{tabular}




\section{SERVICE TRIGGERING}

The ICS service is triggered as described below.

- On the user profile, that is, on all calls from a direct-access profile invoke the ICS service.

- On the combination of two call settings:

- one call settings which is the identity of the incoming trunk, that is, all calls coming from a given trunk marked as an "ICS trunk" invoke the ICS service;

- one call setting which is the access prefix. For example, all calls dialed with a prefix indicating the operator as the chosen longdistance carrier are triggered, and all calls starting with another prefix for another carrier are not.

- On the reception of a call with a given numbering prefix (for example, 36PQ).

The ICS service is not be triggered if the call demands another IN-provided service. For instance, if a call is received from an ICS trunk but the calling user has dialed one of the 800 prefixes, the relevant 800 service is invoked and not the ICS service.

\section{SERVICE PROVISION}

The operator or service provider manages the service provision. The operator or service provider will have access to the service management either via the information system or via a PC with a user-friendly graphics interface running under Windows ${ }^{\mathrm{T} M}$.

Service information is managed using the object-oriented concept. The operator or service provider can apply management methods to service objects. The main man-to-machine commands are the following: Create, Modify, Display, Remove, List.

The service provider will be able to perform management of configuration, data, statistics, administration, and mail. 


\section{SERVICE SUBSCRIPTION}

ICS service provision requires an arrangement between the service provider and the service subscriber. The service subscriber can request to withdraw from the arrangement, or the service provider can also decide to withdraw.

Once the subscription between the service provider and the service subscriber has been arranged, the service provider registers a CallerID profile in the IN platform. This profile comprises the settings relative to the customer-subscribed functions. The service subscriber can modify these settings via the service provider.

\section{$7 \quad$ STATISTICS}

The ICS service provides permanent and on-demand statistical information on:

- call-handling statistics, enabling the operator to measure service usage, such as call type, call time, unsuccessful calls, and so on;

- management statistics, enabling measurement of the Service Management Point (SMP) activity (sessions and commands).

The service offers the following examples of counters for operator statistics:

- number of calls presented to the service;

- number of calls successfully processed by the service;

- number of calls primarily routed to an announcement;

- number of calls primarily routed to an IVR;

- number of calls primarily routed to the called party;

- number of calls primarily routed to an attendant;

- number of calls rejected due to call center overload;

- number of calls with connection to the called party;

- number of calls meeting busy called party or congestion;

- number of calls rerouted to the voice-mail delivery after meeting a busy condition. 


\section{CONCLUSION}

In the context of approaching deregulation, new market players in telecommunications are modifying the value chain. LDNOs need to make money with the traffic they carry for the benefit of end-users who are subscribers of local-loop operators.

The ICS service may become key to allowing LDNOs to check incoming calls and decide to accept or reject the calls. A significant feature of incoming call screening is that it allows new subscriptions to be easily registered, thanks to attendants to whom the calls can be routed. This feature is of utmost importance for newcomers, who might not yet have a fully deployed commercial network. With ICS, they will be able to welcome end-users, especially in the initial phases of launching their network.

ICS may ultimately prove to be a new approach to subscribing an LNDO service, thus allowing LDNOs to capture new subscribers efficiently.

\section{GLOSSARY AND ABBREVIATIONS}

\subsection{Glossary}

\section{Access prefix}

Prefix dialed by the calling user before the $E .164$ number, for example, enabling carrier selection. In France, referred to as $E$ or $16 \alpha \beta(\gamma)$.

\section{Attendant}

Operator's employee working in a call center, in charge of welcoming prospects, targets, and service subscribers.

\section{Back-office profile}

Caller's complementary network-transparent characterization, transmitted from the network to the technical information system.

\section{CallerID}

Full set of basic and complementary caller identifications applying to a caller, including the LineID.

\section{Caller profile}

Information that categorizes a CallerID with respect to the subscription profile, and back-office profile, and which determines the service logic to be applied to the call. 


\section{Calling party category}

Category applying to the line used by the caller, for example, ordinary line, priority line, payphone, and so on.

LineID

Various line identities received in the relevant signaling fields. Component of the CallerID.

\section{Long-distance call}

Calls between two different local areas, or placed to an international destination.

\section{Long-distance network}

Network which routes long-distance calls.

\section{Long-Distance Network Operator, LDNO}

Network operator which handles long-distance calls for its customers. The longdistance network operator uses the ICS service to control the access to the longdistance network. Also referred to as the operator.

\section{Network operator}

Entity which operates network elements and resources.

Nomadic subscriber

Subscriber using the service with identification means which differ from the calling line identity.

\section{Operator}

See Long-Distance Network Operator.

\section{Prospect}

Entity or person known as possibly interested in the long-distance network service.

\section{Remote access}

Access reserved to nomadic subscribers, for which the caller identification is not linked to the line identification.

\section{RemoteID}

Specific caller identification used in case of nomadic subscribers' remote access.

\section{Service logic}

A program which determines how to handle a long-distance call which has triggered the ICS service. For a given caller, there will be one given associated caller profile, which in turn determines one given service logic, with a group of given settings, to be applied to that person's calls.

\section{Service provider}

Entity responsible for dealing with the customer. Also called in French Société de Commercialisation de Services (SCS).

\section{Subscriber}

Entity or person, which or who has subscribed to the long-distance network, possibly through a service provider. 


\section{Subscription profile}

Characterization of a caller encompassing subscription status information and refining it through complementary data.

Subscription status

Categorization of a caller with respect to the subscription process.

\section{Target}

Prospect with whom a commercial contact has already been established.

\subsection{Abbreviations}

$\begin{array}{ll}\text { CDR } & \text { Call Detailed Record } \\ \text { CLI } & \text { Calling Line Identity } \\ \text { CPC } & \text { Calling Party Category } \\ \text { ICS } & \text { Incoming Call Screening } \\ \text { IN } & \text { Intelligent Network } \\ \text { IS } & \text { Information System } \\ \text { IS-C } & \text { Commercial Information System } \\ \text { ISDN } & \text { Integrated Services Digital Network } \\ \text { IS-T } & \text { Technical Information System } \\ \text { IVR } & \text { Interactive Voice Response } \\ \text { LDNO } & \text { Long-Distance Network Operator } \\ \text { PC } & \text { Personal Computer } \\ \text { PLMN } & \text { Public Land Mobile Network } \\ \text { PSTN } & \text { Public Switched Telephone Network } \\ \text { SCP } & \text { Service Control Point } \\ \text { SMP } & \text { Service Management Point } \\ \text { SSF } & \text { Service Switching Function } \\ \text { UPI } & \text { User-Provided Identity } \\ & \end{array}$

\section{BIOGRAPHIES}

\section{P. Bleuse}

In 1980 Patrice Bleuse graduated in electronics engineering from the Ecole Nationale Supérieure d'Electronique de Caen (ENSEEC/ISMRA), after which he obtained a post-graduate management degree from the Institut d'Administration d'Entreprise (IAE), Paris, France.

He spent the next ten years as R\&D director with a French industrial and automotive equipment manufacturer. $\mathrm{He}$ was responsible for designing 
telecommunications equipment such as phonecard boxes and radio terminals, for both industry- and military-class products.

In 1990 he joined Alcatel Telecom as product manager for modems (V/F and baseband) and ISDN-NTs. He then headed the ATM LAN program within the Alcatel Data Networks (ADN) company.

Today, a member of Alcatel Telecom's network systems and product management, Bleuse has worldwide responsibility for Internet-related network solutions for the public switch, the Alcatel E10 system.

\section{B. Vilain}

Bernard Vilain is a 1977 graduate of the Ecole Nationale Superieure des Télécommunications (ENST), Paris, France.

He spent the first seven years of his career at the Centre National d'Etudes des Télécommunications (CNET), the research arm of France Telecom, where he was in charge of product analysis of digital exchanges to prepare the French PSTN for the introduction of ISDN. In 1984, he joined Alcatel Telecom as project manager for pre-intelligent network services on the Alcatel transit node.

At present, his responsibilities cover IN standards activities for Alcatel Telecom. Within the company's network systems and product management organization, he is program manager for intelligent networks and distributed environments; in this capacity he prepares network offerings for IN and mobile product lines worldwide. He was recently nominated chairman for the ITU-T Working Party $2 / 11$ for the 1997-2000 study period, in charge of ISUP, DSS1, and protocol interworking issues. 